\title{
Percepción de la motivación de los directivos intermedios en tres hospitales de la Región del Maule, Chile
}

\author{
Miguel Alejandro Bustamante-Ubilla, Dr en C Econ y Emp, (I) \\ María Carolina del Río-Rivero, M en Des y Compor Organiz, (I) \\ Germán Enrique Lobos-Andrade, Dr en C de Gest, ${ }^{(1)}$ Patricia Isabel Villarreal-Navarrete, Ing Comer. ${ }^{(I)}$
}

\begin{abstract}
Bustamante-Ubilla MA, Del Río-Rivero MC, Lobos-Andrade GE, Villarreal-Navarrete PI. Percepción de la motivación de los directivos intermedios en tres hospitales de la Región del Maule, Chile. Salud Publica Mex 2009;5 I:41 7-426.
\end{abstract}

\section{Resumen}

Objetivo. En este trabajo se diseña un cuestionario y se cuantifican las percepciones de motivación-desmotivación de los jefes intermedios de tres hospitales de la Región del Maule, Chile. Material y métodos. El trabajo de campo se realizó entre septiembre y octubre de 2006, se aplicó un cuestionario con 57 afirmaciones de medición de actitudes que se calificó de acuerdo con una escala tipo Likert de cinco puntos. La población objeto de la investigación fue de 125 profesionales bajo cuya supervisión se encuentran alrededor de 3800 funcionarios. Resultados. Se identificaron I0 variables, cinco motivacionales y cinco desmotivacionales. Entre las primeras destacan vocación y espíritu de servicio; entre las segundas falta de reconocimiento y falta de compromiso. Discusión. Se confirma que tanto las variables motivacionales como las desmotivacionales son esencialmente cualitativas y que las variables económica y de sueldos son menos relevantes y de inferior jerarquía.

Palabras clave: conducta; personal de salud; recursos humanos; motivación; métodos; Chile
Bustamante-Ubilla MA, Del Río-Rivero MC,

Lobos-Andrade GE, Villarreal-Navarrete PI.

Motivation perception measurement of intermediate directors

in three complex hospitals of the Region of the Maule, Chile.

Salud Publica Mex 2009;5 1:417-426.

\section{Abstract}

Objective. In this work, a questionnaire was designed and perceptions of motivation and demotivation of middle managers in three hospitals in the Region del Maule, Chile were measured. Material and Methods. The fieldwork was carried out between September and October, 2006. A questionnaire that included 57 statements to measure attitude was administered and qualified according to a fivepoint Likert-type scale. The population studied included I 25 professionals that supervise roughly 3800 employees. Results. Ten variables were identified, 5 motivational and 5 demotivational. Notable among the motivational variables are vocation and service-oriented spirit; among the demotivational variables are lack of recognition and commitment. Discussion. It is affirmed that both motivational variables as well as demotivational variables are essentially qualitative and that economic and salary variables are less relevant and less hierarchical.

Key words: behavioral research;motivation; human resources; methods; Chile

(I) Facultad de Ciencias Empresariales, Universidad de Talca, Chile.

Fecha de recibido: 16 de febrero de 2009 • Fecha de aprobado: 17 de junio de 2009

Solicitud de sobretiros: Dr. Miguel Alejandro Bustamante-Ubilla. Facultad de Ciencias Empresariales, Universidad de Talca, Chile. Casilla 72I, Talca, Chile.

Correo electrónico:mabu@utalca.cl 
$\mathrm{D}$ urante las últimas dos décadas, los planes del gobierno chileno se han enfocado en modernizar el sistema de salud ${ }^{1,2}$ y abordar sobre todo procesos macroinstitucionales como ejes de acción del Estado. Los esfuerzos se han centrado en la consecución de grados superiores de equidad ${ }^{3}$ mediante el mejoramiento de la gestión de los servicios públicos. ${ }^{4}$ Se definieron ya los establecimientos de autogestión en red, ${ }^{5}$ a los cuales se les exige una gestión moderna, el desarrollo de competencias de su personal, la institución de sistemas de participación, el establecimiento de mejores prácticas y una eficaz gestión de las personas. ${ }^{6,7}$ En consecuencia, se han sentado las bases de un cambio ${ }^{8,9}$ que dio inicio durante el año 2005 y que se encuentra en plena ejecución. ${ }^{10,11}$

En este contexto, el impacto en la Región del Maule, Chile, que integra en su área geográfica a cuatro provincias con una población de un millón de habitantes, ha sido de grandes proporciones. Su sistema de salud, administrado por un servicio de amplia cobertura, es el más complejo del país, tanto por su amplitud geográfica, desde la cordillera hasta el mar, como por su dispersión demográfica y estructura poblacional, sobre todo rural, hasta conformar un complejo estadio para la reforma, cuya aplicación ha recibido múltiples y algunas veces contrapuestas observaciones, relacionadas con la reforma, sus características, el sistema público, la dotación y el sistema de estímulo que promuevan estabilidad. ${ }^{12}$

Sobre la base del tipo de motivaciones mencionadas en el párrafo anterior, surgió entre los directivos de los tres mayores hospitales de la Región del Maule el objetivo de medir la percepción de motivación-desmotivación de los jefes intermedios, descrita en sus diversas fases en este trabajo.

\section{Conceptos en torno de la motivación}

En el campo de la psicología o la administración, la motivación se ha definido de diversas formas, ${ }^{13}$ hasta coincidir en que es la causa del comportamiento de un organismo o bien la razón por la que un organismo lleva a cabo una actividad determinada. Se la relaciona con incentivos e impulsos, así como con la voluntad de lograr objetivos que satisfaga alguna necesidad individual. ${ }^{13}$

Las teorías de proceso conciben la conducta del individuo como el resultado de las determinaciones humanas e identifican las formas en que se activan, dirigen, sostienen y detienen las conductas. Sobresalen, entre ellas, las teorías del establecimiento de metas, expectativas, disonancia cognoscitiva y evaluación cognitiva. ${ }^{14}$

La motivación se origina en el rompimiento de un estado de equilibrio que genera tensión, estimula impul- sos endógenos del individuo e integra "factores capaces de provocar, mantener y dirigir la conducta", ${ }^{15}$ con el propósito de alcanzar grados superiores de homeostasis. Se afirma que la motivación genera una descarga de tensión y liberación del desequilibrio en un comportamiento enfocado en objetivos cuya consecución reduce las tensiones que actúan como impulsoras. ${ }^{14}$

Además, las denominadas teorías del proceso de intercambio social, la teoría de la equidad, balance o equilibrio y la teoría social cognitiva constituyen los pilares para comprender las interacciones sociales que suceden en el lugar de trabajo; esto permite, por una parte, explicar las reacciones de los empleados hacia sus labores y, por otra, asumir la función de su participación dentro la organización. ${ }^{16}$

La teoría del contenido de los dos factores ${ }^{17}$ distingue, en primer lugar, factores motivadores directamente relacionados con la naturaleza del trabajo y los sentimientos positivos hacia él y su contenido. Identifica, en segundo lugar, factores de higiene, que dan contexto al desempeño laboral de los individuos, evitan la insatisfacción y permiten entender el comportamiento humano en los ambientes de trabajo, de tal manera que los factores determinantes de la satisfacción laboral no son los mismos que causan la insatisfacción y que la ausencia de los factores determinantes de satisfacción no conducen en todos los casos a la insatisfacción laboral. ${ }^{18}$

Es sobre esta base conceptual que, para activar la conducta de las personas, se ha sugerido instituir acciones con énfasis en el fortalecimiento de factores que estimulan los aciertos y atenúan el impacto de incongruencias, ${ }^{18}$ se ha propuesto también establecer objetivos, políticas y controles en todas las actividades y hacer efectivas las teorías del contenido, ${ }^{17,19,20}$ del proceso, ${ }^{13,14}$ y del proceso de intercambio social, lo cual ha dado origen a un modelo integrado de motivación que en conjunto permite explicar los elementos de motivación-desmotivación.

\section{Material y métodos}

Se realizó un estudio descriptivo, transversal, observacional y retrospectivo, con un diseño de investigación multivariado. Se diseñó y validó un cuestionario y su aplicación se efectuó mediante un estudio de tipo cuantitativo. Se recogió información demográfica e información de actitudes y el análisis de los datos se llevó a cabo mediante el software estadístico SPSS versión 12.0.

\section{Descripción de la población}

El trabajo de campo se realizó mediante un censo de 125 profesionales directivos. El criterio de inclusión fue que 
constituyeran la línea de mando intermedia de los hospitales complejos, cabeceras de provincia de la región, incluidos los jefes de servicios clínicos, las unidades de apoyo terapéutico, las unidades de apoyo diagnóstico y las jefaturas de servicios administrativos (cuadro I), bajo cuya supervisión se encuentran alrededor de 3800 funcionarios del sector salud de la Región del Maule, Chile. Asimismo, se excluyeron de la selección las cinco direcciones superiores de los hospitales, entre las cuales figuran la dirección general y las subdirecciones médica, administrativa, de operaciones y enfermera coordinadora.

\section{Descripción de los instrumentos utilizados}

Se analizaron diversas teorías de la motivación, se identificaron variables y se elaboró un listado de 154 preguntas en relación con la motivación laboral. Se obtuvo una definición operacional de ellas y se acotaron ciertas dimensiones a fin de conformar ítems representativos que se discutieron en sesiones de grupo focal, destinadas a consensuarlas y sintetizarlas a un número manejable de preguntas que evidenciaran una lógica de sentido interpretable.

Se aplicó la técnica de grupo focal para el análisis de situaciones de motivación en el contexto de la salud. Se propiciaron espacios de reflexión acerca de sus condiciones, posibilidades y consistencia, incluidos técnicas de comunicación, autoinformes y respuestas a estímu- los no estructurados o parcialmente estructurados. Se ratificaron los hallazgos mediante la articulación del lenguaje con los gestos, como expresión de sentimientos en torno de los contenidos de las variables, se aplicaron algunas técnicas de observación del comportamiento manifiesto y reacciones fisiológicas espontáneas de los participantes, por una parte, para la identificación de contenidos, ratificación de componentes relevantes y, por otra, para la definición de los contenidos implícitos en las variables que debía contener el cuestionario.

Los resultados preliminares del cuestionario se mostraron con las mismas palabras de los actores sociales y se evitó la interpretación. En la siguiente fase se clasificaron los temas, se relacionaron las descripciones verbales tal y como las mencionaron los actuantes y se obtuvieron conclusiones para cada ítem.

Se estructuraron las afirmaciones en un cuestionario genérico que consideró preguntas de apertura, transición, claves, término y síntesis. Por último, se efectuó una ratificación grupal del instrumento desarrollado mediante la realización de talleres de retroalimentación, que se complementaron con entrevistas en profundidad con directivos y expertos para conformar los agrupamientos y validar su sentido.

En los talleres se aplicó el método SOPP adaptado, en el cual se le entregó a cada asistente tres papeles de un color y tres de colores diferentes. En los primeros debían escribir tres atributos que motivaban el desarrollo de las labores que realizaban y en los otros aquellos

Cuadro I

Jefaturas intermedias de los hospitales de la Región del Maule, Chile

\begin{tabular}{|c|c|c|c|}
\hline Subdirecciones & $\begin{array}{c}\text { Servicios clínicos y } \\
\text { administrativos }\end{array}$ & Subdirecciones & Servicios clínicos y administrativos \\
\hline Subdirección de Operaciones & $\begin{array}{l}\text { Alimentación } \\
\text { Esterilización } \\
\text { Servicios generales } \\
\text { Equipos médicos } \\
\text { Mantenimiento }\end{array}$ & Servicios clínicos & $\begin{array}{l}\text { Medicina } \\
\text { Pediatría } \\
\text { Cirugía } \\
\text { Obstetricia ginecológica } \\
\text { Cirugía infantil }\end{array}$ \\
\hline Subdirección Administrativa & $\begin{array}{l}\text { Recursos humanos } \\
\text { SOME } \\
\text { Control y presupuesto } \\
\text { Abastecimiento } \\
\text { Informática }\end{array}$ & \multirow{2}{*}{$\begin{array}{l}\text { Unidades de apoyo } \\
\text { terapéutico }\end{array}$} & $\begin{array}{l}\text { Emergencia } \\
\text { Pabellón } \\
\text { UCl } \\
\text { Consultorio adosado } \\
\text { Oncología }\end{array}$ \\
\hline Unidades de Apoyo y Diagnóstico & $\begin{array}{l}\text { Laboratorio } \\
\text { Banco de sangre } \\
\text { Anatomía patológica } \\
\text { Imagenología }\end{array}$ & & $\begin{array}{l}\text { Centro de cáncer gástrico } \\
\text { Especialidades } \\
\text { Unidad asistencial nutricional intensiva } \\
\text { Rehabilitación } \\
\text { Pensionado general }\end{array}$ \\
\hline
\end{tabular}

Fuente: elaboración propia sobre la base de los organigramas y los registros de las unidades administrativas que se indican: Servicio de Orientación Médico Estadístico, SOME; Unidad de Cuidados Intensivos, UCl; Unidad Asistencial Nutricional Intensiva, UANI 
que lo desalentaban. A continuación se agruparon las aportaciones por colores hasta conformar dos grupos, el que representaba elementos de motivación y el correspondiente a los de desmotivación.

En seguida, los participantes se dividieron en dos grupos de trabajo que realizaron una subclasificación de atributos y los ordenaron por "familias" que hacían referencia a un mismo tema. Estos agregados confirmaron cada una de las variables de motivación y desmotivación identificadas en la primera actividad.

En la tercera fase, es decir, la de ratificación de contenidos, se le entregó a cada persona un total de seis votos, tres para cada grupo de variables, que se asignaron de acuerdo con la valoración o significación personal del concepto analizado (se podían asignar uno o los tres votos a un mismo atributo, lo que indicaba la relevancia del factor de motivación o desmotivación). Mediante este procedimiento se generó el efecto de frecuencia y se representó la puntuación de cada variable resultante de la adición de los votos que obtuvieron los atributos que las componían.

\section{Elaboración del cuestionario de motivación-desmotivación laboral}

El cuestionario se estructuró en tres partes, en las cuales se utilizaron las escalas nominal, ordinal, de intervalo y razón. En la primera parte se llevó a cabo la medición de actitudes respecto de la motivación y se aplicó una Likert de cinco puntos a través de 57 afirmaciones. La medición consideró la aplicación de un conjunto de preguntas indirectas cuyos resultados se agruparon para la evaluación de las respectivas dimensiones de motivación y desmotivación. Mediante este procedimiento, los encuestados indicaron que estaban de acuerdo o no con las oraciones, que expresaban actitudes muy positivas o muy negativas respecto del objeto consultado, tras elegir entre cinco respuestas alternativas.

Se establecieron cinco intervalos de clase para la interpretación de la escala Likert. Entre 1.0 y 1.79 puntos el rango "Completamente en desacuerdo" y entre $4.2 \mathrm{y}$ 5.0 puntos el rango "Completamente de acuerdo". Hay que destacar el hecho de que las preguntas 4, 17, 19, 29, $33,38,40$ y 48 eran negaciones a fin de evitar el sesgo en las declaraciones, de tal modo que para el análisis de datos, la escala de cinco puntos fue invertida y la respuesta con número 3 permaneció como neutra.

Las preguntas de la segunda parte del cuestionario permitieron priorizar tanto las variables motivacionales (M) como las desmotivacionales (D) al relacionar las variables y el número de preguntas $(\mathrm{P})$. Como variables de motivación funcionaron las codificadas por $\left(\mathrm{M}_{\mathrm{n}}\right)$, donde $\mathrm{M}_{1}$ es desarrollo personal, $\mathrm{M}_{2}$ vocación, $\mathrm{M}_{3}$ económico,
$\mathrm{M}_{4}$ espíritu de servicio y $\mathrm{M}_{5}$ relaciones humanas. Por su parte, como de desmotivación, las codificadas $\left(D_{n}\right)$ fueron las siguientes: $\mathrm{D}_{1}$ es estilo de administración, $\mathrm{D}_{2}$ falta de reconocimiento, $\mathrm{D}_{3}$ sueldo, $\mathrm{D}_{4}$ falta de compromiso, $\mathrm{D}_{5}$ falta de organización, $\mathrm{D}_{6}$ relaciones interpersonales, $\mathrm{D}_{7}$ falta de recursos, $\mathrm{D}_{8}$ relaciones familiares y $\mathrm{D}_{9}$ ambiente laboral.

La tercera parte del cuestionario tuvo como objetivo jerarquizar las variables de motivación y desmotivación, para lo cual se utilizó una escala ordinal creciente de siete puntos a fin de conocer la importancia y significación de éstas en los entrevistados. En dicha escala los resultados se obtuvieron tras clasificar objetos o arreglarlos en un orden respecto de una variable común. La escala proporciona información en cuanto a la importancia de las variables de motivación-desmotivación. El ranking resultante se consigue mediante una estandarización a la normal de los promedios. Además, con el objetivo de asegurar la sinceridad de las personas al responder y la confidencialidad de la información, se optó por un cuestionario anónimo.

\section{Piloteo del instrumento}

La conducción preliminar del cuestionario se realizó mediante piloteo con una muestra aleatoria de $30 \mathrm{di}$ rectivos de salud. Como criterios de inclusión de los elementos a entrevistar se determinó que formaran parte del nivel directivo superior de los hospitales o que se ubicaran en la tercera línea de mando de la estructura, con lo cual se aseguró que se tratara de directivos pertenecientes a la estructura hospitalaria, pero que no estuvieran incluidos entre los elementos del censo. El criterio de inclusión/exclusión tuvo el sentido de asegurar que en las pruebas preliminares se revisaran la comprensión, el lenguaje, el formato, las instrucciones y las escalas a utilizar, así como el de descartar la existencia de dudas respecto de las preguntas formuladas, lo que evitó influir previamente en los sujetos que se entrevistarían en el censo. Esta preprueba se efectuó en un día durante el mes de septiembre del año 2006 y se la sometió a la opinión de seis académicos expertos.

La validación por expertos permitió asegurar la unidireccionalidad de las proposiciones y ratificar el lenguaje utilizado, así como la redacción y estructura de las afirmaciones.

\section{Procedimiento}

Los propios investigadores llevaron a efecto el trabajo de campo en un lapso de tres semanas en el mes de octubre de 2006. Se elaboró un programa de abordaje a 
las unidades clínicas y administrativas, de tal manera que la aplicación del cuestionario se realizó en forma personalizada en los propios lugares de trabajo y tomó la forma de una entrevista estructurada con el objetivo de obtener, con la mayor certeza posible, información válida y confiable.

Sobre la base del principio de consentimiento informado, los investigadores notificaron a los entrevistados la regulación que existía para el efecto de la aplicación de cuestionarios establecida por el Estado de Chile mediante la Ley $\mathrm{N}^{\circ}$ 17.347. A continuación se solicitó la colaboración de los directivos entrevistados hasta alcanzar la totalidad de las respuestas.

\section{Resultados}

\section{Caracterización demográfica de la población}

La población de estudio se conformó con $80 \%$ de hombres y $20 \%$ de mujeres. En cuanto a su nivel de escolaridad universitario, todos tenían el grado de licenciado y título profesional de 8 a 10 semestres. Sus edades se ubican entre los 30 y 66 años, con una media de 44.6 años. El ingreso laboral al sistema de salud público databa de 1967 a 2006 y 57\% de los entrevistados mostró ingreso posterior al año 1987. La jornada de trabajo de las jefaturas intermedias es la habitual en Chile, con un total de ocho horas diarias de lunes a viernes, además de los turnos que por razones de buen servicio deben realizar. Por último, las jefaturas intermedias alcanzan el segundo nivel de ingresos del sistema hospitalario y se hallan en el rango de 1200 a 2000 dólares mensuales.

Mediante la observación del comportamiento manifiesto y reacciones fisiológicas espontáneas de los participantes se hicieron evidentes diversas expresiones emocionales, entre las cuales figuran las de ánimo, autoconciencia y autocontrol, así como también las de afectividad y empatía relacionadas con los elementos de motivación-desmotivación en el trabajo. Se logró por este medio captar la sintonía emocional, el ritmo social, la postura y la expresión facial en coincidencia con el tenor de los planteamientos expresados.

Entre los factores identificados destacan la lealtad en la organización, las características de honradez e integridad, la responsabilidad en el cumplimiento de las obligaciones, la capacidad de reconocer las necesidades de los usuarios y la de satisfacer sus necesidades, además de la tenacidad para alcanzar objetivos y la manifestación de respeto hacia la organización.

\section{Validez del contenido, el constructo y la convergencia}

Se verificó la validez del contenido sobre la base de la teoría de Herzberg. El diseño del cuestionario consideró factores intrínsecos y extrínsecos que constituyen las nociones básicas de la motivación laboral y que representan los dos componentes del modelo. La validez del constructo se determinó mediante la identificación y definición operativa de las variables constitutivas de motivación-desmotivación, la ratificación se obtuvo a través de grupos focales y la confirmación mediante consulta de expertos.

La validez convergente se determinó mediante el análisis de factores de componentes principales. Se verificaron las correlaciones, las cargas y las varianzas explicadas de cada factor para dar validez a las variables, dimensiones y agregados representativos del presente estudio. Se consideraron las cargas de los componentes respecto de cada factor superior o iguales a 0.5. La validez discriminante se verificó mediante las varianzas explicadas de cada factor en cuanto a la varianza total y su confiabilidad a través del alfa de Cronbach, cuyo índice se ubicara en un rango superior o igual a 0.5 para cada factor.

\section{Factorización de variables}

Con el objetivo de confirmar la estructura del cuestionario, se determinaron los factores mediante un análisis de factores de componentes principales y rotación Varimax, un procedimiento mediante el cual se extrajeron seis factores de acuerdo con la norma eigenvalues superiores a la unidad de Kaiser.

Se consideró pertinente un valor igual o superior a 0.70 de confiabilidad y se verificó que los resultados alcanzados por cada uno de los factores permitieran asegurar índices superiores al valor umbral y se los ubicó en el rango de 0.73 a 0.88 de confiabilidad y la varianza que explica cada factor.

En el cuadro II se reproducen los ítems de los seis factores determinados, el término designado para expresarlos, el número de ítems o preguntas, los respectivos coeficientes de correlación entre los componentes de cada factor y los índices de fiabilidad correspondientes. Este análisis permitió agrupar las variables en conjuntos homogéneos de cinco o seis componentes que, en general, muestran altas cargas (correlación) entre los componentes y el respectivo factor.

El factor 1 (satisfacción laboral) explica la mayor proporción de varianza (14\%) en tanto que el de menor 


\section{Cuadro II}

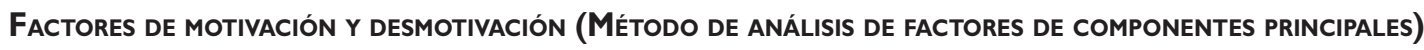

\begin{tabular}{|c|c|c|c|c|c|}
\hline \multicolumn{3}{|c|}{ Factor I: Satisfacción laboral } & \multicolumn{3}{|c|}{ Factor 2: Condiciones del trabajo } \\
\hline Pregunta & Variable & Correlación & Pregunta & Variable & Correlación \\
\hline 10 & Satisfacción personal & 0.84 & 7 & Ascensos & 0.80 \\
\hline 16 & Labor & 0.84 & 12 & Grado servicio & 0.89 \\
\hline 22 & Vocación de servicio & 0.75 & 15 & Remuneración recibida & 0.80 \\
\hline 52 & Ayuda paciente & 0.69 & 38 & Tipo trabajo & 0.35 \\
\hline 53 & Espíritu de servicio & 0.90 & 50 & Entorno laboral & 0.59 \\
\hline & Coeficiente alfa & 0.88 & & Coeficiente alfa & 0.79 \\
\hline & Varianza explicada & 0.14 & & Varianza explicada & 0.11 \\
\hline \multicolumn{3}{|c|}{ Factor 3: Falta de reconocimiento } & \multicolumn{3}{|c|}{ Factor 4: Compromiso organizacional } \\
\hline Pregunta & Variable & Correlación & Pregunta & Variable & Correlación \\
\hline 17 & Comunicación & 0.70 & 30 & Compromiso & 0.69 \\
\hline 31 & Ambiente laboral & 0.41 & 32 & Beneficios laborales & 0.86 \\
\hline 34 & Críticas & 0.83 & 42 & Desorden organizacional & 0.58 \\
\hline 43 & Burocracia & 0.58 & 44 & Compromiso colegas & 0.52 \\
\hline 47 & Dirección & 0.52 & 51 & Lealtad laboral & 0.46 \\
\hline & Coeficiente alfa & 0.73 & & Coeficiente alfa & 0.74 \\
\hline & Varianza explicada & 0.09 & & Varianza explicada & 0.07 \\
\hline \multicolumn{3}{|c|}{ Factor 5: Falta de recursos } & \multicolumn{3}{|c|}{ Factor 6: Convivencia laboral } \\
\hline Pregunta & Variable & Correlación & Pregunta & Variable & Correlación \\
\hline 26 & Recursos & 0.74 & II & Relaciones laborales & 0.80 \\
\hline 39 & Recursos humanos & 0.20 & 23 & Relaciones humanas & 0.68 \\
\hline 40 & Materiales & 0.88 & 27 & Clima laboral & 0.26 \\
\hline 48 & Incentivo monetario & 0.47 & 33 & Desarrollo profesional & 0.27 \\
\hline \multirow[t]{3}{*}{54} & Desempeño & 0.27 & 37 & Nuevos conocimientos & 0.36 \\
\hline & Coeficiente alfa & 0.70 & & Coeficiente alfa & 0.71 \\
\hline & Varianza explicada & 0.06 & & Varianza explicada & 0.05 \\
\hline
\end{tabular}

Nota: se utilizó el método de análisis multivariado de factores de componentes principales

Se aplicó el software de análisis estadístico SPSS versión 12.0

varianza explicada es el factor 6 (convivencia laboral) (5\%). Los factores incorporan un total de 30 variables que muestran índices de correlación, desde el más alto que registra la variable espíritu de servicio con índice de 0.90 hasta el más bajo que posee la variable clima laboral con índice de 0.26 .

Por lo regular, los factores se observan bien conformados, con excepción del factor 3 (falta de reconocimiento) que incluye el componente ambiente laboral, al parecer distante del sentido que define a este factor, pese a que evidencia una carga de 0.41 , y el factor 4 (compromiso organizacional) que incluye la variable desorden organizacional con una carga factorial de 0.58 . Es factible, en consecuencia, suponer que a futuro nuevos estudios pudieran redefinir la variable, según sea el contenido de los ítems, al eliminar algunos componentes y, al final, añadir otros más relacionados que aseguren la unidimensionalidad de los respectivos factores.

\section{Percepción de la motivación-desmotivación}

Con el objetivo de comparar los resultados obtenidos en las provincias de Linares, Curicó y Talca, se estandarizaron las medias y se obtuvieron números exentos del efecto de las respectivas varianzas. El resultado de este procedimiento permitió determinar 
valores estándar comparables que se ubican, desde el punto de vista estadístico, en un rango homogéneo (-3 $a+3)$. Los valores estandarizados con signo negativo aparecen cuando la media de la variable es menor a la media general del segmento y, en el caso de los de signo positivo, si la media de la variable se halla por encima de la media general.

En primer lugar (cuadro III), la variable vocación de servicio resulta ser la más relevante y alcanza una media estandarizada que fluctúa entre 1.84 y 1.18 puntos de la estándar para los directivos intermedios de los hospitales de Curicó y Linares, respectivamente, en tanto que la menos motivacional fue la variable económico relacionada a su vez con la variable desmotivacional sueldo.

En segundo lugar se ubica espíritu de servicio y su mayor impacto se presenta en el hospital de Linares, con 1.15 puntos de la estándar y el valor más bajo se observa en el hospital de Talca con sólo 0.19 puntos. Se advierte además que para los directivos del hospital de Curicó la variable desarrollo personal, que de variadas formas incorpora los componentes desarrollo profesional y nuevos conocimientos, es una de las que menos altera la motivación y es levemente superior en la percepción de los directivos de los hospitales de Linares y Talca.

Entre las variables de menor impacto motivacional, la variable relaciones humanas fluctuó entre -0.41 puntos para los directivos del hospital de Curicó y 0.18 puntos en el hospital de Linares, en tanto que en el hospital de Talca alcanzó un índice cercano a 0. Por su parte, las variables ambiente laboral y falta de recursos presentaron, en general, valores negativos y cercanos a 0 . Por último, el factor económico resulta ser el de menor impacto motivacional para los profesionales del hospital de Curicó y alcanzó sólo - 0.71 puntos y apenas 0.03 puntos de la estándar en el hospital de Linares.

En relación con las variables que generan desmotivación (cuadro IV), los directivos intermedios del hospital de Talca consideran las variables falta de reconocimiento, falta de compromiso, relaciones interpersonales, falta de organización, sueldo, relaciones familiares y ambiente laboral como indicativas de desmotivación; sus valoraciones estándar son positivas y se hallan por encima de la media general; en cambio, las variables estilo de administración y falta de recursos mostraron escasa dispersión de sus medias estandarizadas y se ubicaron levemente por debajo de la media general del segmento, lo que confirma el carácter desmotivacional de dichas variables.

Una percepción complementaria, pero diferente, se observa entre los directivos del hospital de Linares, quienes perciben las variables con valoraciones estándar negativas, con excepción de las variables relaciones laborales y sueldo. Por su parte, los directivos del hospital
Cuadro III

Percepción de la motivación (VAlores estandarizados)

\begin{tabular}{|c|c|c|c|}
\hline Variables motivacionales & Linares* & Curicó* & Talca* \\
\hline Vocación & 1.18 & 1.84 & 0.40 \\
\hline Desarrollo personal & 0.34 & 0.18 & 0.30 \\
\hline Espíritu de servicio & 1.15 & 0.70 & 0.19 \\
\hline Relaciones humanas & 0.18 & -0.41 & 0.01 \\
\hline Económico & 0.03 & -0.71 & -0.02 \\
\hline
\end{tabular}

* Medias estandarizadas

Nota: se aplicó el software de análisis estadístico SPSS versión I2.0

\section{Cuadro IV \\ Percepción de la desmotivación (VAlores estandarizados)}

\begin{tabular}{lccc}
\multicolumn{1}{c}{ Variables desmotivacionales } & Linares* & Curicó & Talca* \\
Falta de reconocimiento & -0.28 & 0.20 & $\mathrm{I} .91$ \\
\hline Falta de compromiso & -0.81 & 0.14 & $\mathrm{I} .41$ \\
\hline Relaciones interpersonales & 0.14 & -0.52 & 0.46 \\
\hline Falta de organización & -0.52 & -0.06 & 0.29 \\
\hline Sueldo & 0.03 & -0.47 & 0.21 \\
\hline Relaciones familiares & -0.06 & 0.92 & 0.11 \\
\hline Ambiente laboral & -0.03 & -0.05 & 0.10 \\
\hline Estilo de administración & -0.41 & 0.07 & -0.70 \\
\hline Falta de recursos & -0.07 & -0.16 & -0.19
\end{tabular}

* Medias estandarizadas

Nota: se aplicó el software de análisis estadístico SPSS versión I2.0

de Curicó perciben cuatro variables por encima de la media ( 0.07 para la variable estilo de administración y 0.20 para la variable falta de reconocimiento) y cinco de ellas por debajo de la media (-0.52 para la variable relaciones interpersonales y -0.05 para la variable ambiente laboral), lo que las ratifica también como desmotivacionales.

El análisis por variables hace posible afirmar que la falta de reconocimien to es altamente desmotivacional con un índice de 1.91 puntos de percepción en el hospital de Talca y con -0.28 puntos estándar en el hospital de Linares por debajo de la media de las percepciones. En segundo lugar, la variable falta de compromiso alcanza 1.41 en el hospital de Talca y, en contraste, en el hospital de Linares obtiene -0.81 puntos y se ubica por debajo de la media de las percepciones. Por otra parte, relaciones familiares alcanza su mayor índice en el hospital de $\mathrm{Cu}$ ricó con 0.92 puntos y desciende a sólo 0.11 puntos en el 
hospital de Talca. En Linares presentó valores cercanos a 0 y se confirmó como causal de desmotivación.

El estilo de administración permite discriminar levemente entre los distintos hospitales de la región. Para las jefaturas del hospital de Curicó la percepción se ubica en 0.07 puntos estándar, en tanto que en los hospitales de Linares y Talca llegó a - 0.41 y - 0.7 puntos, respectivamente. En este mismo sentido, la variable falta de organización logra entre los directivos del hospital de Talca 0.29 puntos; no obstante, para los directivos del hospital de Linares el valor de la media estandarizada correspondió a sólo -0.52 puntos estándar.

\section{Jerarquización de variables de motivación-desmotivación}

Conforme lo señalado en un párrafo anterior, la interpretación de los valores estándar con signo negativo pone en evidencia que la media de la variable es menor a la media general del segmento analizado y que un valor positivo le confiere una mayor importancia relativa.

$\mathrm{Al}$ jerarquizar las variables de motivación (cuadro V), se observa que la variable vocación se halla en la primera jerarquía en los tres hospitales y alcanza el mayor indicador (1.85 puntos) en el hospital de Curicó. Le sigue la variable espiritu de servicio que ocupa la segunda jerarquía en los hospitales de Curicó (0.70) y Linares (0.10).

CuadroV

JERARQUIZACIÓN DE LAS VARIABLES DE MOTIVACIÓNDESMOTIVACIÓN (VALORES ESTANDARIZADOS)

\begin{tabular}{|c|c|c|c|c|c|c|}
\hline Variables de motivación & OJ* & Curicó $\neq$ & & Linares $^{\ddagger}$ & OJ & Talca $^{\ddagger}$ \\
\hline Vocación & I & 1.85 & I & 0.42 & I & 0.40 \\
\hline Espíritu de servicio & 2 & 0.70 & 2 & 0.10 & 3 & 0.19 \\
\hline Desarrollo personal & 3 & 0.18 & 3 & 0.05 & 2 & 0.30 \\
\hline Relaciones humanas & 4 & -0.41 & 4 & -0.06 & 4 & 0.01 \\
\hline Económico & 5 & -0.72 & 5 & -0.26 & 5 & -0.02 \\
\hline Variables de desmotivación & OJ* & Curicó $\neq$ & & Linares $^{\ddagger}$ & OJ & Talca $^{\ddagger}$ \\
\hline Estilo de administración & 1 & 0.37 & 5 & -0.29 & 2 & -0.01 \\
\hline Falta de reconocimiento & 2 & 0.01 & 3 & 0.05 & 5 & -0.17 \\
\hline Falta de compromiso & 3 & -0.03 & I & 0.17 & 1 & 0.28 \\
\hline Falta de organización & 4 & -0.07 & 4 & 0.04 & 3 & -0.03 \\
\hline Sueldo & 5 & -0.16 & 2 & 0.10 & 4 & -0.04 \\
\hline
\end{tabular}

En la tercera jerarquía, la variable desarrollo personal destaca en Talca con 0.3 puntos, mientras la variable relaciones humanas consigue la cuarta posición (-0.41, -0.06 y 0.01) por debajo de la media de los datos del segmento, lo cual evidencia baja prioridad relativa entre los directivos de los tres hospitales. Por último, la variable económico presenta la media estandarizada de más bajo nivel jerárquico con una media estandarizada de sólo - 0.72 puntos en el hospital de Curicó.

La jerarquía de las variables de desmotivación (cuadro V) muestra distintas prioridades en los tres hospitales; empero, la variable estilo de administración resulta ser la más importante en el hospital de Curicó (0.37), en tanto que en el hospital de Talca (-0.01) es desmotivacional. La variable falta de reconocimiento produce desmotivación entre los directivos de los hospitales de Linares y Curicó, mientras que en Talca se le asigna escasa relevancia, con una media estandarizada de sólo - 0.17 puntos. Le sigue la variable falta de compromiso (0.28) en el hospital de Talca, en comparación con la obtenida en el hospital de Curicó (-0.03).

De manera complementaria, la variable falta de organización se percibe como de desmotivación en el hospital de Linares, con una media de 0.04 puntos y para los hospitales de Curicó y Talca es de -0.07 y -0.03 puntos, respectivamente, lo que ubica a dichas percepciones en una jerarquía levemente inferior. Por último, la variable sueldo se constituye en la variable menos importante para los directivos intermedios de los tres hospitales. Sin perjuicio de ello, para el personal intermedio del hospital de Linares esta variable es relativamente importante, con una media estandarizada que registró 0.1 puntos estándar.

\section{Discusión}

La motivación laboral es un tema que desafía la gestión en las organizaciones. Algunos estudios han identificado factores causales de ausencia de motivación en unidades clínicas específicas o han determinado grados de motivación y satisfacción laboral en hospitales de especialidad..$^{21}$ Además, se han efectuado estudios de satisfacción laboral de los trabajadores de atención primaria ${ }^{22}$ y estudios en torno de las condiciones laborales, características individuales, vida laboral y familiar que provocan efectos motivacionales. Por último, se han determinado los grados de confort, eficiencia y satisfacción laboral de usuarios internos en establecimientos de salud ${ }^{23}$ lo que ha puesto de manifiesto la relevancia de este tipo de investigaciones.

Como se infiere de tales trabajos, la medición de los niveles de motivación-desmotivación de los man- 
dos directivos intermedios de hospitales complejos que presenta este protocolo es clave para la gestión de organizaciones de salud; asimismo, se analiza la dinámica motivacional, sobre todo en periodos de cambio estructural, como es el que actualmente vive el sistema de salud de Chile.

Con todo, un análisis detallado de los hallazgos permite afirmar que vocación y espíritu de servicio evidencian cuán endógena es la motivación, por una parte, ya que sus fundamentos se ubican en la persona misma, sus sentimientos y su sensibilidad hacia el trabajo y, por añadidura, hacia quienes se sirve, todo lo cual revela que en lo más profundo de la persona el acto de servir es satisfactorio. Dicha interpretación se confirma en estudios de prácticas de directivos de salud pública que citan dimensiones influyentes, como motivación, liderazgo, reciprocidad y participación; ${ }^{24-26}$ y donde llama la atención la variable libertad como elemento clave de la motivación que se relaciona con conductas tales como compromiso y disfrute del trabajo. Es por ello que el principio de secuencia propuesto por Maslow ${ }^{19}$ parece evolucionar en la persona del directivo, quien no centraría su atención precisamente en "sus necesidades", sino que el individuo se sostiene en sus capacidades, se ajusta a los estímulos intrínsecos y alcanza autosatisfacción al servir a los demás.

Es por ello que el modelo teórico de Herzberg ${ }^{17}$ destaca la importancia del reconocimiento por pares y supervisores, al sugerir que las significaciones personales del esfuerzo, la importancia del resultado en el trabajo y el papel alcanzado en la función generan en el espacio laboral altos niveles de satisfacción. Este argumento explica otro de los hallazgos de esta investigación, que identifica las variables falta de reconocimiento y falta de compromiso como las de mayor jerarquía generadoras de desmotivación.

Consecuente con lo anterior, la variable falta de reconocimiento $0^{1,19}$ se ubica como la de mayor desmotivación y ello sugiere que los directivos parecen esperar algo que está más allá de una compensación pecuniaria y que más bien esperarían que ésta satisfaga alguna necesidad del tipo ego/autoestima/reconocimiento, ${ }^{19}$ lo cual pone de manifiesto el valor de los intangibles en la motivación. ${ }^{26}$

Por su parte, la variable falta de compromiso ${ }^{1,14}$ evidencia que la omisión de reconocimiento explícito se interpreta de forma negativa, genera tensión en los directivos y, tal vez por ello mismo, explica la propensión a compensar su insatisfacción con niveles inferiores de compromiso y desempeño. Al parecer, esta conducta permitiría a los directivos obtener un seudoestado de equilibrio de las expectativas desempeño-compensacio- nes esperadas que se acerca bastante al propiciado por el modelo de motivación de las expectativas de Vroom. ${ }^{14}$

Estos hallazgos exteriorizan la naturaleza de lo que los directivos consideran motivacional-desmotivacional. Las variables de motivación (vocación y espiritu de servicio) son esencialmente intangibles, se hallan en lo profundo de la persona y su identificación en el sector de la salud contribuye al entendimiento de una cierta lógica de sentido que ayuda a los directivos a la satisfacción de sus propias necesidades. En posición contraria, las variables generadoras de desmotivación (falta de reconocimiento y falta de compromiso), también de naturaleza intangible, avanzan por encima de la trampa de lo material (dinero, ropa, comida, etc.).

Como efecto de lo descrito, las variables económico y sueldos se confirman como las menos relevantes, comprueban los factores higiénicos de Herzberg ${ }^{17}$ y se valoran como variables de contexto, de modo tal que resultan extrínsecas al desempeño directivo. Sin embargo, los efectos de estas variables sobre el clima organizacional son innegables y se han reconocido diversas dimensiones (estructura, responsabilidad, recompensa, desafío, relaciones, cooperación, estándares, conflictos e identidad), dado que posicionan las relaciones recompensas e identidad como relevantes y altamente valoradas.

Se vislumbra la opción de elaborar un Plan de motivación que incluya el diseño de un nuevo sistema de calificación, formación de comités, programas de participación del empleado y mejorías sustanciales en la gestión administrativa de los centros asistenciales. Dichas acciones, de forma implícita, las delinearon los diversos grupos humanos analizados, para reforzar aquellos factores que resultaron desmotivacionales, $y$ pudieran instituirse a mediano y largo plazos.

Además, es perfectamente posible actuar sobre las variables económico y sueldo porque pueden ser generadoras de motivación, tras considerar el grado en que se encuentren cubiertas las necesidades de subsistencia y según sea el valor otorgado que la dimensión seguridad alcance en relación con el estándar de satisfacción a que aspiren los directivos.

Por último, los resultados reflejan la situación motivacional de los jefes de servicios intermedios de tres hospitales complejos de una región y validan el cuestionario utilizado y la aplicación de la metodología. Sin embargo, se prevé que futuras investigaciones podrían incrementar el número de observaciones, el número de ítems y los niveles de confiabilidad mediante el estudio del sistema de salud en su totalidad constituido por 28 servicios territoriales que coordinan las respectivas redes asistenciales de salud de Chile. 


\section{Referencias}

I. Giaconi J. Readecuación del sistema de salud chileno. Chile: Administración y Economía U.C. 1991;7:16-19.

2. Giaconi J. Políticas y organización del sector salud: década de los ochenta. Transición a la Democracia y Salud. Chile: María Inés Romero, 1992:167-204

3. Hollstein R, Vega J, CarvajalY. Desigualdades sociales y salud: nivel socioeconómico y mortalidad infantil en Chile, 1985-1995. Rev Med Chile 1995;126:333-340.

4. Hernández S, Sandoval H, Delgado Y. Monitoreo y seguimiento de la reforma: determinación de línea basal de equidad en el financiamiento y protección financiera. Chile: Departamento de Estudios y Desarrollo, Superintendencia de Salud, 2006.

5. Ley $N^{\circ}$ 19.937, Reforma del Sector Salud, Chile.

6. Jadue L, Delgado I, Sandoval H.Análisis del nuevo modelo de salud de la encuesta CASEN 2000. Rev Med Chile 2004;132:750-760.

7. Giaconi J. Futuro de los sistemas de salud. Rev Univer 1993;XL:10-12.

8. Subramanian V, Delgado I, Jadue L. Inequidad de ingreso y autopercepción de salud: un análisis desde la perspectiva contextual de las comunas chilenas. Rev Med Chile 2003; |31:321-330.

9. Salinas H, Erazo M, Reyes A. Indicadores de gestión de servicios de salud públicos y asignación de recursos desde el ministerio de salud de Chile. Rev Med Chile 2004;132(I2):I532-I542.

I0.Vicente B, Kohn R, Valdivia S, Rioseco P, Torres S. Plan nacional de salud mental en Chile: 10 años de experiencia. Rev Panam Salud Publica 2007; I. II. Salinas J, Cancino A, Pezoa S, Salamanca F, Soto M.Vida Chile 19982006: resultados y desafíos de la política de promoción de la salud en Chile. Rev Panam Salud Pública 2007;2I (2/3):136-1 44.

12. FENPRUSS. Mensaje de S.E. la presidenta de la república con el que inicia un proyecto de ley que modifica el decreto con fuerza de ley $n^{\circ} \mathrm{I}$, de 2005, del Ministerio de Salud, la ley $n^{\circ}$ 19.490; se delegan facultades para fijar y modificar las plantas de personal que indica y otros beneficios para el personal de los servicios de salud, 25 de septiembre de 2006. Disponible en: http://www.fenpruss.cl.

13. Madsen K. Teorías de la motivación: un estudio comparativo de las teorías modernas de la motivación. 2da ed. Buenos Aires: Paidós, 1972. I4. Deci L. Effects of externally mediated reward on intrinsic motivation.J Appl Psychol 197I;18:105-II5.
15. Arana W. Motivación. Revista Ser Humano y Trabajo 2005. Disponible en: www.sht.com.ar/archivo/liderazgo/motivacion.htm. 16. Adams J.Toward an undestanding of inequity. J Abnor Soc Psychol 1963;76:422-436.

17. Herzberg F, Mausner B, Snyderman B. The motivation to work. 2nd ed. New York: John Wiley and Sons, 1959.

18.Vansteenkiste M, Simons J, Lens W, Sheldon K, Deci E. Motivating learning, performance, and persistence: the synergistic effects of intrinsic goal contents and autonomy-supportive contexts.J Personal Soc Psychol 2004;87:246-260.

19. Maslow A. Motivation and personality. Nueva York: Harper, 1954:419. 20. Mclelland D. The achieving society. New York: McGraw Hill; Princeton, Van Nostrand, 1961:514.

21. Zúñiga-Vallejos R. Estudio de la motivación y producción de un servicio de cirugía. Hospital II Essalud,Talara-Piura-Perú. Disponible en: rizuva[arroba]mixmail.com

22. Fernández-San Martín MI, Moinelo-Camporro A, VillanuevaGuerra A,Andrade RC, Rivera-Teijido M, Gómez-Ocaña JM, et al. Satisfacción laboral de los profesionales de atención primaria del área 10 del Insalud de Madrid. Rev Esp Salud Publica 2000 [periódico en la Internet].Abr [consultado 2009 abr 08]; 74(2): Disponible en: http://scielo.isciii.es/scielo.php?script=sci_arttext\&pid=SI I35$57272000000200005 \& \operatorname{lng}=e s \& n r m=i s o$.

23. Delgado-Rigel T, Saco-Mendez S, Paredes-Calcina S. Grado de satisfacción laboral del usuario interno en los establecimientos de salud de la red Quispicanchi-Acomayo-Cusco. SITUA 2003;12(23):36-43. 24. Bastidas-Mera E, Estrada-Alava J, Rivera Vallejo G. Clima organizacional y su incidencia en la calidad de los servicios públicos: Cedenar, Empopasto y Telenariño. [Consultado 2009 abril I7]. Disponible en: http://www. monografias.com/trabajos / 4/servpublicos/servpublicos.shtml\#CREAT 25. Presno-Labrador MC. Condiciones organizativas y género en el sistema gerencial del área de salud. La Habana. [trabajo para optar por el título de Master en Salud Pública]. Cuba: Facultad de Salud Pública, 1997. 26. Segredo-Pérez AM, Miranda-Reyes D. Clima organizacional en salud pública. Consideraciones generales/Organizational Environment in Public Health. General Considerations. Cuba: Escuela Nacional de Salud Pública de Cuba, 2004. 